\title{
Nickel Decorated Single-Wall Carbon Nanotube as CO Sensor
}

\author{
Ngangbam Bedamani Singh, Barnali Bhattacharya, Utpal Sarkar* \\ Department of Physics, Assam University, Silchar, Assam. \\ Email: ${ }^{*}$ utpalchemiitkgp@yahoo.com \\ Received October $1^{\text {st }}, 2013$; revised November $3^{\text {rd }}, 2013$; accepted November $10^{\text {th }}, 2013$ \\ Copyright (C) 2013 Ngangbam Bedamani Singh et al. This is an open access article distributed under the Creative Commons Attribution \\ License, which permits unrestricted use, distribution, and reproduction in any medium, provided the original work is properly cited.
}

\begin{abstract}
Based on spin-polarized density functional theory (DFT) calculations, the interaction between nickel cluster decorated single-wall carbon nanotube (CNT) and CO molecule has been investigated. DFT calculations are performed with generalized gradient approximation (GGA) using Perdew-Burke-Ernzerhof (PBE) functional. Interaction of CNT and cluster induces spin polarization in the CNT. Nickel decorated CNT has a large magnetic moment of $4.00 \mu_{B}$ which decreases to $0.10 \mu_{\mathrm{B}}$ when $\mathrm{CO}$ molecule is absorbed to it. Such a drastic reduction in magnetization may be detected by SQUID magnetometer. Hence by measuring magnetization change, CNT-cluster system may be used as gas detectors. The charge transfer between the systems has been discussed through Mulliken charge analysis for different orientations of the adsorbed CO molecule. We observed that CNT-cluster system acts as electron donor and CO molecule acts as electron acceptor in this study.
\end{abstract}

Keywords: Nickel Cluster; Carbon Nanotube; CO Sensor; DFT; Magnetic Moment

\section{Introduction}

Carbon nanotubes (CNT) can be used in a number of applications such as scanning probes [1], nanoelectronic devices [2], chemical sensors [3] etc. Sensing gas molecules like $\mathrm{CO}, \mathrm{CO}_{2}, \mathrm{NO}, \mathrm{NH}_{3}, \mathrm{O}_{2}, \mathrm{~N}_{2}, \mathrm{H}_{2}, \mathrm{CH}_{4}$ are vital in a number of fields such as environmental monitoring, agricultural, medical, industrial applications, space missions, control over chemical processes, etc. [3,4]. Nanoscale sensors based on single-wall carbon nanotubes have high sensitivity and fast response time for detecting $\mathrm{NO}_{2}$ and $\mathrm{NH}_{3}$ [5]. Understanding the nature of interaction between the adsorbed molecule and CNT is important to fully exploit the potential application of CNT as sensors. Searching for the ways to enhance the adsorption energies is a novel strategy for the gas sensing use of CNT. To create active sites for the adsorption of molecule on the intrinsical inert surface of the CNT, many techniques have been employed, such as, introducing defects on the walls of the CNT [6], embedding or doping foreign atoms [7]. One of the most common methods in sensing technology is the surface adsorption of metal clusters on CNT sidewalls [8]. It is reported that decoration of

*Corresponding author.
SWCNT with transition metals exhibits higher sensitivity than the pristine CNTs [8]. The sensitivity of platinum and gold decorated CNTs increases by an order of magnitude for $\mathrm{NH}_{3}$ and $\mathrm{NO}_{2}$ detection. In the present study, Ni clusters have been chosen as they are used as catalysts and also exhibit strong adsorption properties to a number of molecules [9]. CO molecule has been considered because of its role as pollutants and toxicants.

\section{Calculation Method}

Based on generalized gradient approximation (GGA), spin polarized density functional calculations are performed using norm-conserving pseudopotential. The single-zeta basis set has been used and the exchange correlation part of density function is treated with PerdewBurke-Ernzerhof (PBE) function. All the calculations are performed by using siesta-3.1 package [10]. The full geometry relaxation was performed until the forces on the atoms are less than $0.04 \mathrm{eV} / \AA$ and the mesh cut-off value was set at 300 Rydberg. Tetragonal unit cell of 25 $\times 25 \times 12.92 \AA^{3}$ is employed for the periodic boundary conditions. Semiconducting $(5,0)$ zigzag SWCNT with supercell containing 60 carbon atoms have been used. 
We place $\mathrm{Ni}_{4}$ cluster of tetrahedron structure at the surface of the nanotube, then, the $\mathrm{CO}$ molecule is placed at different sites above and around the $\mathrm{Ni}_{4}$ decorated CNT. The binding energy $\left(E_{b}\right)$ of CO molecule on the nanotube is calculated as:

$$
E_{b}=E_{(\mathrm{CNT}+\text { Cluster }+\mathrm{CO})}-E_{(\mathrm{CO})}-E_{(\mathrm{CNT}+\text { Cluster })}
$$

where $E_{(\mathrm{CNT}+\text { Cluster) }}, E_{(\mathrm{CO})}$ and $E_{(\mathrm{CNT}+\text { Cluster }+\mathrm{CO})}$ are the total energies of the cluster decorated carbon nanotube, $\mathrm{CO}$ molecule and carbon nanotube-cluster-CO system respectively. From the Mulliken population analysis, charge transfer between the adsorbed molecule and system $(\mathrm{CNT}+$ Cluster $)$ is analysed.

\section{Results and Discussions}

The optimized structure of pristine $(5,0)$ SWCNT has a diameter of $4.17 \AA$. The $\mathrm{C}$-C bond length along the nanotube axis (denoted as $\mathrm{B}_{\|}$in Figure 1) is $1.42 \AA$ and the other bond aligned partially perpendicular to the nanotube axis $\left(\mathrm{B}_{\perp}\right)$ is $1.47 \AA$. When the $\mathrm{CO}$ molecule is adsorbed on the sidewalls of the pristine $(5,0)$ carbon nanotube, $\mathrm{C}-\mathrm{C}$ bonds in the hexagonal rings near the adsorbed $\mathrm{CO}$ molecule deforms. The $\mathrm{B}_{\|}$and $\mathrm{B}_{\perp}$ bond in the hexagonal ring close to the adsorbed molecule becomes $1.38 \AA$ and $1.52 \AA$ respectively. As we put $\mathrm{Ni}_{4}$ cluster on the surface of the $\mathrm{CNT}$, the bond $\mathrm{B}_{\|}$near the cluster become $1.46 \AA$ and the bond $\mathrm{B}_{\perp}$ near the cluster is $1.52 \AA$. When we place the $\mathrm{CO}$ molecule on the $\mathrm{Ni}_{4}$ decorated $\mathrm{CNT}$, the tetrahedron structure of the cluster becomes
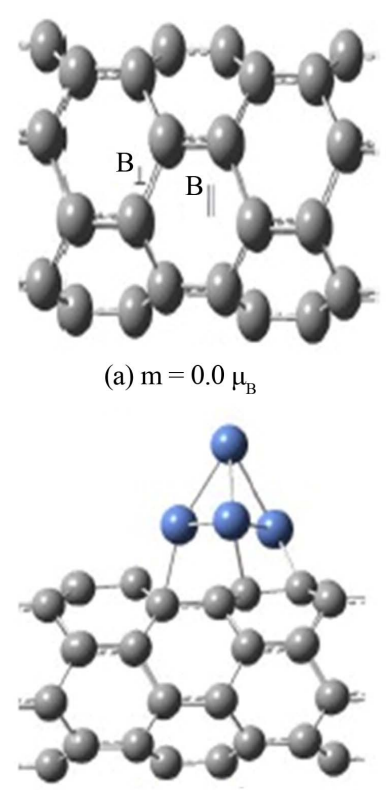

(c) $\mathrm{m}=4 \mu_{\mathrm{B}}$ (a) $\mathrm{m}=0.0 \mu$ (b) $\mathrm{m}=0.0 \mu_{\mathrm{B}}$

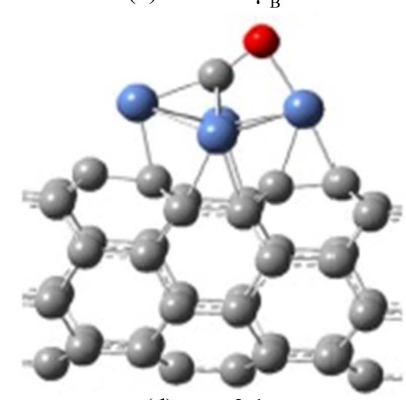

(d) $\mathrm{m}=0.1 \mu_{\mathrm{B}}$

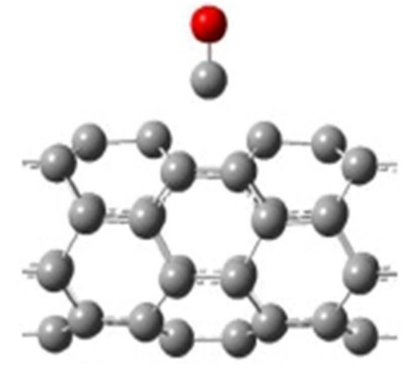

Figure 1. Structure and observed magnetic moments, $m$ of (a) pristine $(5,0)$ SWCNT (b) CO adsorbed on $(5,0)$ SWCNT (c) $\mathrm{Ni}_{4}$ decorated $(5,0) \mathrm{SWCNT}$ (d) CO adsorbed on the $\mathrm{Ni}_{4}$ decorated $(5,0)$ SWCNT. distorted. The most stable geometry has CO molecule near the centre of the cluster with $\mathrm{C}$ atom towards the cluster. Change in the $\mathrm{C}-\mathrm{C}$ bond in the hexagonal ring near the adsorbed molecule is also observed and $\mathrm{CO}$ is at a height of $2.62 \AA$ from the CNT. The $\mathrm{B}_{\|}$bond close to the adsorbed molecule is $1.44 \AA$ and the $\mathrm{B}_{\perp}$ bond is found to be $1.52 \AA$.

The binding energy of $\mathrm{Ni}_{4}$ cluster on the $(5,0) \mathrm{CNT}$ is $-7.97 \mathrm{eV}$. The $-\mathrm{ve}$ value indicates the stability of the cluster decorated CNT, which is required for using them as sensor. The binding energy of the $\mathrm{CO}$ molecule on the pristine CNT is $-11.70 \mathrm{eV}$ and the binding energy on the $\mathrm{Ni}_{4}$ decorated CNTs is $-16.44 \mathrm{eV}$. We observed that the binding energy of $\mathrm{CO}$ is enhanced when the CNT is decorated with $\mathrm{Ni}_{4}$ cluster, a favorable feature for sensors. $\mathrm{CO}$ molecule is an electron attracting molecule with a net positive charge at the oxygen end and an electron affinity of $1.33 \mathrm{eV}$ [11]. Hence, $\mathrm{CO}$ withdraws electrons from the CNT-cluster system. The Mulliken population analysis also reveals that electrons are transferred from the CNTcluster system to the $\mathrm{CO}$ molecule. The charges on the $\mathrm{C}$ and $\mathrm{O}$ atoms of the $\mathrm{CO}$ molecule are $-0.57 \mathrm{e}$ and $-0.29 \mathrm{e}$ respectively and a total charge of $-0.84 \mathrm{e}$ is transferred to the $\mathrm{CO}$ molecule from the CNT-cluster system. The magnetic moments of the structures are presented in Figure 1. The bare $\mathrm{Ni}_{4}$ cluster has a magnetic moment of $6.00 \mu_{\mathrm{B}}$. The pristine CNT and CO adsorbed CNT have zero magnetic moment. On placing the $\mathrm{Ni}_{4}$ at the surface of the non-magnetic CNT, the structure has a net magnetic moment of $4.00 \mu_{\mathrm{B}}$. This magnetic moment is contributed by the Nickel cluster. When $\mathrm{CO}$ is adsorbed on the cluster decorated CNT, we observed that the net magnetic moment suddenly decreases to $0.10 \mu_{\mathrm{B}}$. This sudden drop in the magnetic moment is due to the transfer and rearrangements of electrons between the adsorbed $\mathrm{CO}$ and CNT-cluster system.

\section{Conclusion}

Based on first principle calculations, the possibility of using Nickel cluster decorated single-wall carbon nanotube as CO sensor has been investigated. DFT calculations are performed with generalized gradient approximation (GGA) using Perdew-Burke-Ernzerhof (PBE) function. Mulliken charge analysis shows that the adsorbed $\mathrm{CO}$ molecule acquires electronic charge from the cluster decorated SWCNT. Because of strong CNTcluster interaction, spin polarization is induced in the CNT. Nickel decorated CNT has a large magnetic moment of $4.00 \mu_{\mathrm{B}}$, which changes abruptly to $0.10 \mu_{\mathrm{B}}$ when $\mathrm{CO}$ is attached to it. So adsorption of CO molecule diminished the magnetic property of the system $\left(\mathrm{Ni}_{4}\right.$ decorated SWCNT). This abrupt change in magnetization on $\mathrm{CO}$ adsorption may be detected, hence suggesting the possibility of using CNT-cluster as gas detectors. 


\section{Acknowledgements}

Dr Utpal Sarkar would like to acknowledge, the support from SHARCNET Canada for providing computational facilities for this research work, and Prof Paul W Ayers, Department of Chemistry, McMaster University, Hamilton, Canada for his help in various ways.

\section{REFERENCES}

[1] H. Dai, J. H. Hafner, A. G. Rinzler, D. T. Colbert and R. E. Smalley, "Nanotubes as Nanoprobes in Scanning Probe Microscopy," Nature, Vol. 384, No. 6605, 1996, pp. 147-150. http://dx.doi.org/10.1038/384147a0

[2] S. J. Tans, A. R. M. Verschueren and C. Dekker, "RoomTemperature Transistor Based on a Single Carbon Nanotube," Nature, Vol. 393, No. 6680, 1998, pp. 49-52. http://dx.doi.org/10.1038/29954

[3] J. Kong, N. R. Franklin, C. Zhou, M. G. Chapline, S. Peng, K. Cho and H. Dai, "Nanotube Molecular Wires as Chemical Sensors," Science, Vol. 287, No. 5453, 2000, pp. 622-625. http://dx.doi.org/10.1126/science.287.5453.622

[4] S. Santucci, S. Picozzi, F. D. Gregorio, L. Lozzi, C. Cantalini, L. Valentini, J. M. Kenny and B. Delley, " $\mathrm{NO}_{2}$ and $\mathrm{CO}$ Gas Adsorption on Carbon Nanotubes: Experiment and Theory," Journal of Chemical Physics, Vol. 119, No. 20, 2003, pp. 10904-10910. http://dx.doi.org/10.1063/1.1619948

[5] P. Pannopard, P. Khongpracha, M. Probst and J. Limtrakul, "Gas Sensing Properties of Platinum Derivatives of
Single-Walled Carbon Nanotubes," Journal of Molecular Graphics and Modelling, Vol. 28, No. 1, 2009, pp. 62-69. http://dx.doi.org/10.1016/i.jmgm.2009.04.005

[6] J. Andzelm, N. Govind and A. Maiti, "Nanotube-Based Gas Sensors-Role of Structural Defects," Chemical Physics Letters, Vol. 421, No. 1-3, 2006, pp. 58-62. http://dx.doi.org/10.1016/j.cplett.2005.12.099

[7] L. Bai and Z. Zhou, "Computational Study of B- or NDoped Single-Walled Carbon Nanotubes as $\mathrm{NH}_{3}$ and $\mathrm{NO}_{2}$ Sensors," Carbon, Vol. 45, No. 10, 2007, pp. 2105-2110. http://dx.doi.org/10.1016/j.carbon.2007.05.019

[8] Q. Zhao, M. Nardelli, W. Lu and J. Bernholc, "CarbonNanotube-Metal Cluster Composites: A New Road to Chemical Sensors?" Nano Letters, Vol. 5, No. 5, 2005, pp. 847-851. http://dx.doi.org/10.1021/n1050167w

[9] M. T. Reetz, R. Breinbauer, P. Wedemann and P. Binger, "Nanostructured Nickel-Clusters as Catalysts in [3+2] Cycloaddition Reactions," Tetrahedron, Vol. 54, No. 7, 1998, pp. 1233-1240.

http://dx.doi.org/10.1016/S0040-4020(97)10221-6

[10] P. Ordejon, E. Artacho and J. M. Soler, "Self-Consistent Order-N Density-Functional Calculations for Very Large Systems," Physical Review B, Vol. 53, No. 16, 1996, pp. 10441-10444. http://dx.doi.org/10.1103/PhysRevB.53.R10441

[11] K. M. A. Refaey and J. L. Franklin, "Endoergic IonMolecule-Collision Processes of Negative Ions. III. Collisions of Ion $\mathrm{O}_{2}, \mathrm{CO}$ and $\mathrm{CO}_{2}$," International Journal of Mass Spectrometry and Ion Physics, Vol. 20, No. 1, 1976, pp. 19-32. http://dx.doi.org/10.1016/0020-7381(76)80029-0 